\title{
The Euclidean nature of color space
}

\author{
JOZEF COHEN \\ University of Illinois, Champaign, Illinois 61820 \\ and \\ THOMAS P. FRIDEN \\ The University of New Mexico, Albuquerque, New Mexico 87131
}

\begin{abstract}
All modern theorists use affine geometry as a basis for the vector manifold representing color sensations. Angles and vector lengths have no meaning in affine geometry, and Euclidean vector addition does not apply. All color theories and color specification systems are but transformations in affine space. Color sensations are presumed to be isomorphic to affine geometry alone but both mathematical proof and empirical demonstration show the existence of a transformation for color vectors from affine to Euclidean space. When so transformed to Euclidean space, color vector lengths and direction cosines are invariant under rotation of reference axes; the configuration is stable absolutely, and color vectors add as force vectors in mechanics. New concepts are developed for the Euclidean color space and relationships to the older concepts of affine space (as luminance) are demonstrated.
\end{abstract}

We present a prelimiary report on the Euclidean character of the accepted vector representation of color senstations. Further, we demonstrate the hitherto unknown invariant color vector lengths and invariant direction cosines (angles) between color vectors-the invariant property of Euclidean space only.

All modern color theorists surmise the color manifold to be described by affine space alone. "If these laws about colors, valid according to experience," wrote Erwin Schrödinger in 1920, "are compared with the axioms which must be assumed for vectors drawn from a point in order to esbablish the affine geometry of such a vector pencil, complete agreement is found. Accordingly, the manifold of colors is a tridimensional system of affine structure solely. All special relations in the field of color (sensation) can be made unusually lucid by a vector pencil as a model, so that each color (sensation) corresponds to a single vector ... This model is convenient and even essential, but it possesses a certain danger. We must confine ourselves to the affine relationships, not the metric (Euclidean) relationships which are totally inapplicable."

Affine geometry was developed in the late 19th century; see Weyl (1922). When all definitions and theorems involving length and angle are eliminated from Euclidean geometry, the residue is affine geometry; thus, Euclidean geometry distinguishes circles from ellipses but affine geometry does not.

All color manifolds are based on the "gauged spectrum." This is an $\mathrm{n} \times 3$ Matrix A, where columns represent three arbitrary primaries (in this paper, independent primaries) and rows represent three energy amounts (one for each primary) necessary to match unit amounts of $n$ spectral colors distributed throughout the

We acknowledge the resolute help and superb interrogation of Constantine Trahiotis. We are profoundly beholden to Harold W. Hake. visible spectrum. The three primaries are also represented by rows-those with entries 100,010 , and 001 , so that these three rows form an identity matrix. The remaining rows have one negative entry. The astonishing empirical fact of color vision, and the basis of all color theory, is the relationship $\mathrm{AT}=\overline{\mathrm{A}}$ where $\mathrm{T}$ is any matrix representing a homogeneous linear transformation and $\bar{A}$ is another $n \times 3$ matrix representing a new set of color mixture functions with three new primaries.

In general, Matrix A (and its homogeneous linear transforms) exist in affine space. This is seen immediately when a transform involves only one primary, for in any row, the value for that primary changes as expected, but so also the values for the remaining two unchanged primaries. With respect to Matrix A, vector lengths and angles between vectors (and, of course, angles between axes) have no meaning; they change with every transformation, bringing deformation upon deformation, and in general, the shape of an object in one reference frame has only an affinity for the shape of the same object in the next reference frame-hence the name "affine."

This affine anamorphosis provides unlimited color theories and color specification systems, each based on another affine transformation. When any Matrix $\overline{\mathrm{A}}$ is plotted by columns, so-called "fundamental sensation curves" are obtained. The limitless plots and projections are different and unshapen, and superimposed on each is the construct of luminance, tristimulus values, trichromatic coefficients, and ratio plots on the chromaticity diagram.

Schrodinger's definitive statement of isomorphism between affine geometry and color sensations, like all assertions relating existing phenomena to mathematical/physical models, is but an affirmation of 
faith. Schrodinger's statement is unqualified absolutely, but it still cannot deny unconditionally a "window" where the color vectors of affine space may pass to the color vectors of Euclidean space. We shall know that color vectors have made this passage when, by definition, the identical Matrix $R^{*}$ (the symmetrical matrix of scalar products between vector pairs) can be computed from any A or $\bar{A}$; see Weyl (1948). We have demonstrated the passage, in fact, for we have proved the general existence of $\mathrm{R}^{*}$ and we have computed an invariant $\mathrm{R}^{*}$.

We observe, at the beginning, that tristimulus values are the sums of cross-products and therefore suggestive of direction cosines; they are not direction cosines, however, for they are in affine space where line lengths and angles are meaningless. Then, we define three new concepts: (1) intratristimulus values, the tristimulus values between the primaries of a single set. The $3 \times 3$ intratristimulus Matrix $M$ is defined $A^{\prime} A^{\prime}=M$. (2) autotristimulus values, the tristimulus values of primaries with themselves. Autotristimulus values are the diagonal entries in M. (3) intertristimulus values the tristimulus values of one set of primaries on a second set of primaries. The $3 \times 3$ intertristimulus Matrix $M_{i}$ is defined $\mathrm{A}^{\prime} \overline{\mathrm{A}}=\mathrm{M}_{\mathbf{i}}$.

We have proved that $\mathrm{AM}^{-1} \mathrm{~A}^{\prime}=\mathrm{R}^{*}$, where $\mathrm{R}^{*}$ is invariant for any $\mathrm{A}$; the proof will appear elsewhere. We have computed a $63 \times 63 \mathrm{R}^{*}$ matrix from the Stiles-Burch (1959) $63 \times 3$ matrix A, with primaries at $444.4 \mathrm{~nm}, 526.3 \mathrm{~nm}$ and $645.2 \mathrm{~nm}$-the data given in absolute energy. We have also computed a 63 x $63 \mathrm{R}^{*}$ from these same data transformed to primaries at ${ }^{-}$ $400.0 \mathrm{~nm}, 500.0 \mathrm{~nm}$, and $597.0 \mathrm{~nm}$. The $\mathrm{R}^{*} \mathrm{~s}$ are identical.

The $\mathrm{R}^{*}$ matrix, specifying color vectors in Euclidean space, is the eternal constant of color vision-and contains all information concerning the human visual apparatus for color sensation. Indeed, $\mathrm{R}^{*}$ does possess remarkable properties, and proofs will be given elsewhere. Here, we discuss its most obvious properties.

The matrix $\mathrm{R}^{*}$ can produce all $\mathrm{M}^{1}$ matrices, all $\mathrm{M}_{\mathrm{i}}^{-1}$ matrices, all $\mathrm{M}$ matrices, and all $\mathrm{M}_{\mathrm{i}}$ matrices-for all sets of primaries. To find any $\mathrm{M}^{1}$ or $\mathrm{M}$, locate rows and columns for the same primaries in $\mathrm{R}^{*}$; the $3 \times 3$ matrix so formed is $M^{-1}$, and its inverse is $M$. To find any $M_{i}^{-1}$ or $M_{i}$, locate rows for the first set of primaries and columns for the second set of primaries in $\mathrm{R}^{*}$; the $3 \times 3$ matrix so formed is $\mathrm{m}_{\mathrm{i}}^{-1}$ and the inverse of its transpose is $\mathbf{M}_{\mathbf{i}}$.

The matrices $M^{-1}$ and $M_{i}^{-1}$ provide the passage of $A$ matrices and tristimulus values in affine space to scalar products (in $\mathrm{R}^{*}$ ) in Euclidean space. Given any A or A matrix, multiply by the appropriate $\mathrm{M}^{\mathbf{1}}$ matrix to obtain scalar products on its own primaries, or by the appropriate $\mathbf{M}_{\mathbf{i}}^{-1}$ matrix to obtain scalar products on new primaries. Similarly, given any set of tristimulus values, multiply by the appropriate $\mathrm{M}^{-1}$ matrix to obtain scalar products on its own primaries, or by the appropriate $\mathbf{M}_{\mathbf{i}}^{-1}$ to obtain scalar products on new primaries. Given any three columns of $\mathrm{R}^{*}$, corresponding to any three primaries, multiply by the appropriate $M$ to obtain any $\bar{A}$ matrix with new primaries.

Note that $\mathrm{R}^{*}$ provides the transformation matrices and the color mixture data to be transformed, for all sets of primaries.

The matrix $\mathrm{R}^{*}$ may be factored to a Matrix F, so that $\mathrm{FF}^{\prime}=\mathrm{R}^{*}$; see, for example, Thurstone (1935). In addition, the transformation Matrix $\mathrm{M}^{-1}$ (or $\mathrm{M}_{\mathrm{i}}^{-1}$ ), taken from the appropriate entries in $\mathrm{R}^{*}$, may be similarly factored so that $G^{\prime}=M^{1}$. Then, by proofs to be given elsewhere, $A G=F$, so that $F$ can be obtained directly from A.

An infinite number of $F$ matrices may be obtained from $\mathrm{R}^{*}$, each representing an orthogonal or oblique transformation of reference axes in Euclidean space. An oblique $F$, that is, a configuration with an oblique reference frame, is not equivalent to an A matrix in affine space. An oblique $F$ matrix represents the same invariant color vector representation but with oblique axes. An A matrix has no vector length or angular information. (Note: By proofs to be given elsewhere, we show that an orthogonal $\mathrm{F}$ matrix is transformed to an $\mathrm{A}$ matrix by the appropriate T matrix; see above).

From $\mathrm{R}^{*}$, we have computed an orthogonal $\mathrm{F}$ with principal axes. All eigenvectors are found equal to unity, that is, $F^{\prime} F=I$; this is true of all $F$ matrices obtained by orthogonal rotation. In any orthogonal $\mathrm{F}$, row vectors represent spectral color sensations (vector length equal to the root of sums of squares) and scalar products are the cross products of any two rows.

Since vector lengths and direction cosines are invariant under orthogonal rotation (or oblique rotation), the configuration is absolutely stable. Color vectors add as ordinary Euclidean vectors, and color mixture is exactly analagous to the addition of force vectors in mechanics.

We have plotted the spectral vectors in three-space, to find a closed color domain with three long, nonorthogonal canonical vectors at $444.4 \mathrm{~nm}, 533.3 \mathrm{~nm}$, and $597.0 \mathrm{~nm}$. Vector length is not luminance (see below) but rather a measure of luminous power, a new construct. We have questioned: "Which set of three primaries, of all sets, has minimum luminous sufficiency, that is, requires least energy to match all spectral colors?" Autotristimulus values, one for each primary, measure this energy-but autotristimulus values are in affine space and the three must be evaluated simultaneously. Accordingly, we asked our computer to compare the three autotristimulus values for every set of three primaries-and it selected our own canonical vectors as minimal, those with maximum length. These luminous power peaks have been variously and roughly described by workers in applied colorimetry and 
illumination; see, for example, Thornton (1972).

The best axes position, or best $F$, has yet to be found. Plots of the original Munsell color chips are suggestive immediately. We seek correlates of brightness, chroma, and hue within the Euclidean configurationCartesian coordinates, polar coordinates, or cylindrical coordinates.

This invariant color space is based entirely on well established color-mixture data. Further assumptions are unnecessary.

Finally, we consider the man-made construct of luminance. Luminance, of course, exists in affine space and is thought to be a function of subjective brightness; that relationship is highly questionable. In the invariant Euclidean model, the brightness function will be determined, as it should be, by empirical plot.

Nevertheless and for completeness, we have demonstrated the transfer of luminance from affine space to Euclidean space, again by proofs to be recorded elsewhere. Consider a row vector $A_{\mathbf{L}}$ comprised of the three luminosity coefficients associated with the primaries of Matrix $A$ in affine space; $A_{L}$ is not a row of A. Then $A_{L}$ may be transformed to a row vector of an orthogonal $F$ (a row of $F, F_{L}$ ) by the relationship $A_{L} G^{-1}=F_{L}$. Note that this is not the same transform carrying $A$ into $F$. Luminance then appears as Vector $L$ in Euclidean color space, similar in every respect to all other vectors. We are now uncertain of its precise position, but we are confident of its correspondence to a real desaturated green.

Continuing our exercise with the luminance vector, we have passed one axis of an orthogonal frame through Vector L (representing classical luminance), and the remaining two axes determine an orthogonal plane (representing classical chrominance). The spectral vectors project onto Axis $\mathrm{L}$, and their projections are proportional to the standard luminosity function. The perplexing question concerning the additivity of luminance may now be answered definitively: luminances are vector projections and add as any vector projections in Euclidean space. The spectral vectors also project onto the chrominance plane, forming a closed three-lobed locus passing through all four quadrants; the vector projections add as force vectors on a plane. Of course, the vector lengths in three space are still equal to luminous power and still add as force vectors in three space. This configuration is not equivalent to CIE; the CIE system is in affine space, and vector lengths have no meaning.
David L. MacAdam $(1953,1954)$ is a precursor to the work reported here. As early as 1941, he located orthogonal color-mixture functions in affine space; in our terminology, he defined orthogonal functions as $\mathrm{A}^{\prime} \mathrm{A}$ $=$ I. From time to time, orthogonal color mixture functions have been useful in colorimetry. Our work has shown, however, that orthogonal color-mixture functions define a Euclidean space as well as existing in affine space. MacAdam's color-mixture functions in affine space are precisely equivalent to our orthogonal $\mathrm{F}$ matrices in Euclidean space. So soon as the Euclidean space is realized, then the elegant invariant matrix $R^{*}$ may be computed, invariant vector lengths and direction cosines become evident, the vector configuration becomes stable under rotation, orthogonal and oblique frames may be introduced, color vectors add as force vectors, and the concept of luminous power emerges-all meaningless in affine space.

In 1860 and long before Erwin Schrödinger condemned color vectors affine space alone, James Clerk Maxwell wrote of his insights concerning the domain of color sensation: "In this way, every colour in nature will be represented by a line drawn through the origin, whose direction indicates the quality of the colour, while its length indicates its quantity ... It appears therefore that if colours are represented in quantity and quality by the magnitude and direction of straight lines, the rule for the composition of colours is identical with that for the composition of forces in mechanics."

\section{REFERENCES}

MacAdam, D. L. Dependence of color-mixture function on choice of primaries. Journal of the Optical Society of America, 1953, 43, 533-538.

MacAdam, D. L. Orthogonal color-mixture functions. Journal of the Optical Society of America, 1954, 44, 713-724.

Maxwell, J. C. On the theory of compound colours, and the relations of the colours of the spectrum. Philosphical Transactions of the Royal Society of London; 1860, 150, 57-84.

Schrödinger, E. Grundlinien einer Theorie der Farbenmetrik im Tagessehen. Annalen der Physik, 1920, 368, 397-456, 481-520.

Stiles, W. S., \& Burch, J. M. N.P.L. colour matching investigation: final report. Optica Acta, 1955, 6, 1-26.

Thornton, W. A. Three-color visual response. Journal of the Optical Society of America, 1972, 62, 457-459.

Thurstone, L. L. The vectors of mind. Chicago: University of Chicago Press, 1935.

Weyl, H. Space-time + matter. New York: Dutton, 1920.

$\mathrm{Weyl}, \mathrm{H}$. The classical groups: Their invariants and representations. Princeton, N. J: Princeton University Press, 1938.

(Received for publication November 13, 1974.) 